\title{
Development of the framework for disaster mitigating information sharing platform and its application to a local government
}

\author{
T. Suzuki ${ }^{1} \&$ Y. Hada $^{2}$ \\ ${ }^{1}$ Interdiciplinary Graduate School of Medical and Engineering, \\ University of Yamanashi, Japan \\ ${ }^{2}$ Institute of Industrial Science, the University of Tokyo, Japan \\ (Previously, National Research Institute for Earth Science and Disaster \\ Prevention, Japan)
}

\begin{abstract}
For the purpose of reducing disaster damage by applying information sharing technologies, the research on disaster reduction using crisis-adaptive information sharing technologies commenced in July 2004, as a three-year joint project. In this project, the disaster mitigating information sharing platform, which is effective for disaster mitigation activities, especially for local governments, is developed. This paper describes the results of the three-year project, focusing on the framework of the platform and its application to a local government.

Keywords: information sharing, disaster, response, local government.
\end{abstract}

\section{Introduction}

It is well known that the lack of information sharing led to an increase in damage in past notable disasters, such as the Hyogoken-nanbu earthquake of 1995 in Japan. This lesson has not necessarily been reflected in disaster response activities, even in recent disasters due to a heavy rainfall occurring every year in various parts of the country.

For the purpose of reducing disaster damage by applying information sharing technologies, the research on disaster reduction using crisis-adaptive information sharing technologies commenced in July 2004 as a three-year joint project, composed of a government office and agency, national research institutes, 
universities, lifeline utilities, a NPO and a private company [1,2]. In this project, the disaster mitigating information sharing platform (stated as the DiMIS platform below in this paper), which is effective in disaster response activities, especially for local governments, was developed.

In this project, various information systems and information tools were developed and the research on the standardization of information contents processed through the platform was also carried out. A prototype of the platform was built by integrating an individual system and tool. Then, the platform was applied to a field test for verification. This paper presents the summary of the three-year project, focusing on the framework of the platform and its application to a local government as a field test.

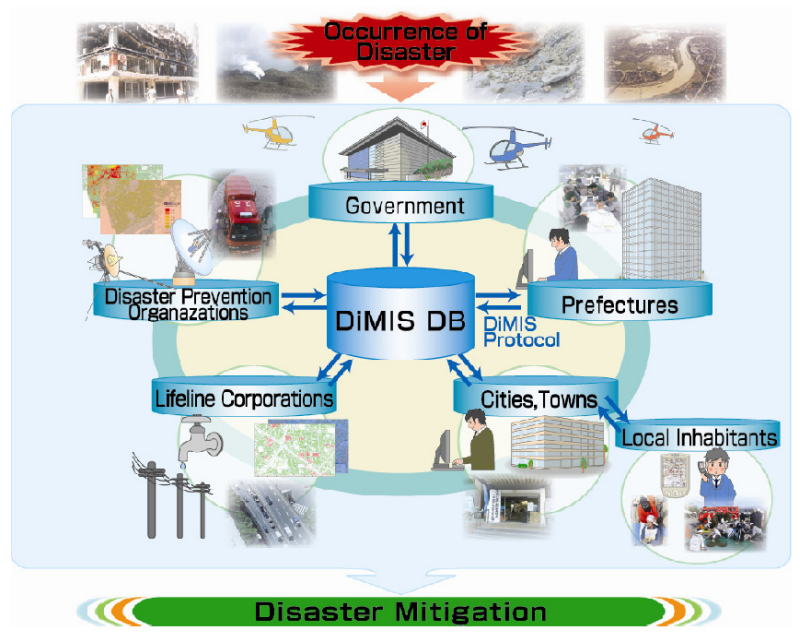

Figure 1: Schematic illustration of the DiMIS platform.

\section{Definition of the DiMIS platform}

In this project, the DiMIS platform is defined as the framework for establishing liaison among different information systems in different governmental agencies and public institutions at the time of disaster response [3]. Figure 1 illustrates a schematic representation of the DiMIS platform. The framework is divided into two different categories, i.e., a category on an information engineering basis and that on a social science basis. The former is represented by the communication protocol, the information sharing database and application software in conformity to the protocol. The latter, on the other hand, is represented by the content of the information and its distribution system. Thus, in other words, the DiMIS platform constitutes the framework regarding both information systems and information content. 


\section{Framework of information systems}

\subsection{Protocol (MISP)}

The communication protocol of the DiMIS platform is the communication procedure to access the DiMIS database. As the prototype of the DiMIS platform, MISP (Mitigation Information Sharing Protocol) was established in the project. XML is used as the data expression and GML is adopted as the geographical data expression in MISP. WFS is adopted as the protocol for database searching and management. SOAP is used as a standardized form of the Web service. Thus, MISP is mainly composed of international standards. The original function of dynamic registering regarding data structure is added to MISP as an extensive function.

Different information systems for supporting disaster response activities, with various database systems and GIS engines, have already been introduced to local governments and public institutions. Therefore, the rigorous standardization of information systems cannot be an expedient approach to establishing liaison among different disaster information systems. Thus, the authors propose a generous unification of various disaster information systems through the information sharing protocol based on MISP.

\subsection{DiMIS database (DaRuMa)}

The DiMIS database is the database in conformity to MISP. The prototype of this database is built on MySQL, an existing relational database, and it was named as DaRuMa. When an application program accesses this database using MISP, it can access MySQL through the protocol processor. It has both the functions of a database server and of a communication server. The access to the database is controlled according to an information sharing rule. In order to enable easier access to DaRuMa from existing information systems, library software that enables the conversion of various data formats is prepared. The concept of the database is illustrated in Figure 2 and the framework of the information systems is summarized in Figure 3.

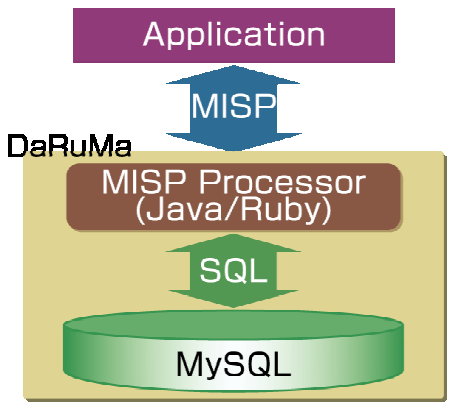

Figure 2: The DiMIS database (DaRuMa).

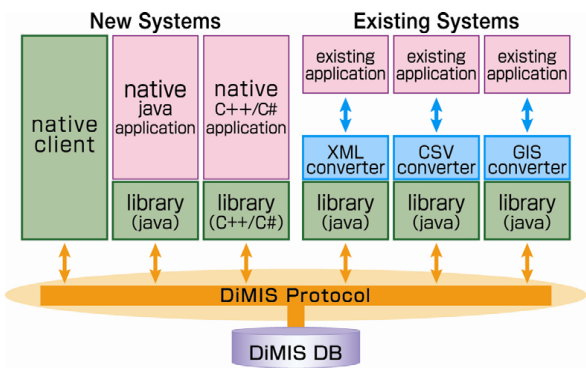

Figure 3: Framework of information systems in the platform. 


\section{Framework of information content}

As a framework for making different information systems communicate with each other, not only "a framework of information systems" explained in the preceding chapter but also "a framework of the content of information" is important. A local government such as a city or a town needs to share various information with local inhabitants, lifeline utilities, disaster prevention organizations such as the police station, the fire station, the Self-Defence Force, hospitals, and the upper local government (prefecture), when a large disaster occurs. In this project, examinations are given on the kind, the form and the accuracy of information required at the time of disaster in the time process. Thus, the investigation was carried out based on a questionnaire and an interview with local government employees who have experienced disaster response activities in actual disasters. Such investigation was conducted in Mitsuke city, Kashiwazaki city, Nagaoka city and Niigata prefecture for the 2004 Niigataken-Chuetsu earthquake and in Mitsuke city for the 2004 Fukushima-Niigata heavy rainfall disaster. It was also carried out in Takamatsu city for the 2004 high tide by typhoon No. 16 and for the 2004 flood by typhoon No. 23, and in Fukuoka city for the 2005 Fukuoka-ken Seiho-oki earthquake and for the 2003 heavy rainfall disaster.

The result of the investigation was arranged and summarized as the database for shared information necessary for disaster response activities. Such information is correspondent with the disaster mitigating information. Based on the database, the information flow diagram was drawn for individually classified information groups. In addition, the information processing flow for individually classified information groups was also summarized [4].

Figure 4 shows a schematic diagram demonstrating the research on the framework of information contents. The table for shared information, in which information items are classified systematically, was made first. Then, an XML schema for each shared information datum was built. It describes the structure of information datum and every information system can access DaRuMa to register, acquire or search datum using the schema, which uses the DiMIS protocol, MISP.

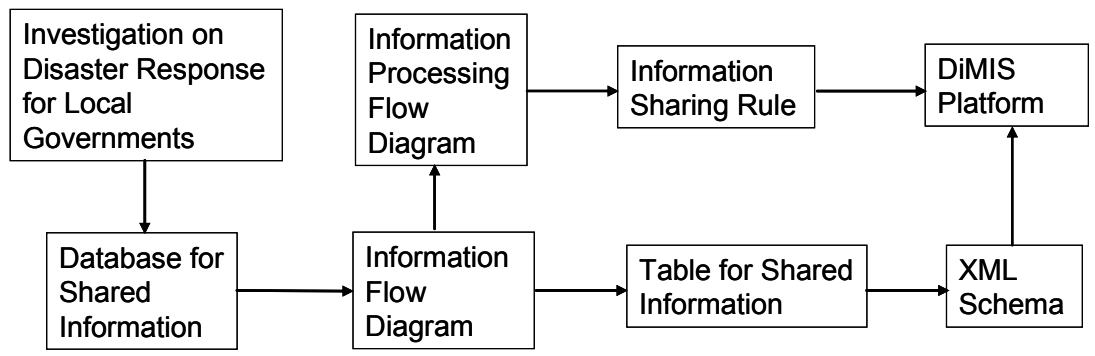

Figure 4: Approach to build a framework of information content. 


\section{Research on efficient utilization of the DiMIS platform}

In order to demonstrate concretely the effectiveness of the DiMIS platform, information systems, tools or applications, which are capable of making efficient use of the shared information, were developed. In this section, the authors introduce some examples representing such information systems and tools developed in the project.

\subsection{Information sharing system supporting disaster response management in a local government}

The disaster response management system for a local government was developed. The system takes important roles not only in information sharing and decision making for disaster response in a local government, but also in communication with various information systems both inside and outside the local government as a client system of the DiMIS database, DaRuMa. The system is a Web application designed to reflect the investigation on disaster response for local governments introduced in section 4 (Figure 5). For the purpose of applying this system to the field test, a prototype system was developed as the disaster response management system for Mitsuke city, Niigata Prefecture, Japan [5].
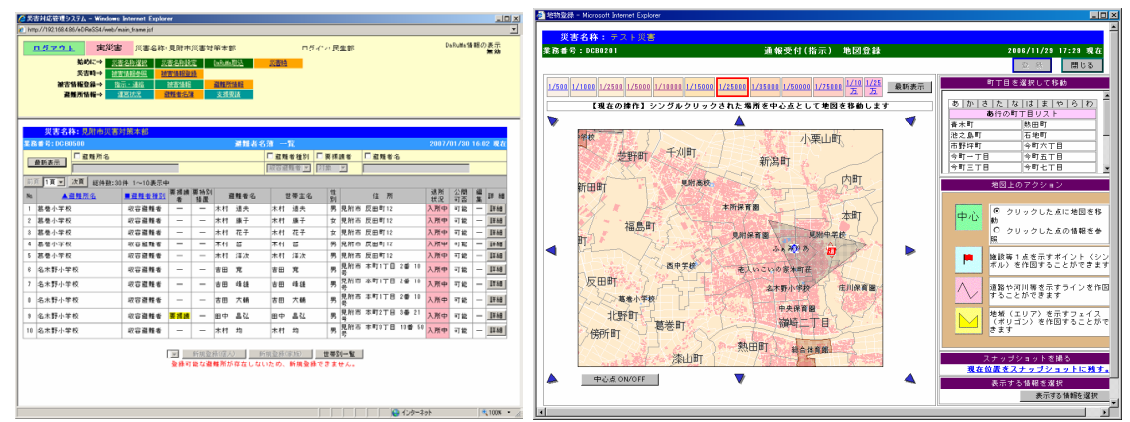

Figure 5: The disaster response management system for a local government.

\subsection{Information sharing system for lifeline utilities}

An examination was given on the information which lifeline utilities are able to supply and need to acquire in a disaster, in the workshop organized by the Cabinet Office, the National Police Agency, the Ministry of Land, Infrastructure and Transport, Tokyo Electric Power Company, Tokyo Gas Company, Nippon Telegraph and Telephone East Corporation (NTT East), and National Research Institute for Earth Science and Disaster Prevention. Based on the examination, the information which lifeline utilities need to acquire through the DiMIS platform in a disaster was summarized as an information table [6]. 
The prototype of an information sharing system for lifeline utilities, which deals with the above information, was developed in the project. Then, the information sharing experiment was carried out, in which the seismic disaster prevention information system of the Tokyo Gas Company was connected with DaRuMa through the prototype of the information sharing system.

\subsection{Viewer system and GUI guidelines}

In order to achieve smooth information sharing, the GUI guidelines were established. The method of data input and display on screens for PDAs, cellular phones, and large displays was developed and it was summarized as the guidelines. Then, the prototype of a viewer system and a few data collecting tools were developed according to the guidelines.

\subsection{Simulators}

Several simulation systems were developed. They receive the necessary input data from DaRuMa, then conduct a simulation and register the simulation outputs to DaRuMa. The simulation system for optimum operation of regional fire fighting power was developed, as a client simulator of the DiMIS platform (Figure 6a).

The evacuation simulator was also developed as a client system of the platform, in consideration of the characteristics of evacuation activities in a local area or in a local core facility. This system obtains disaster information through the DiMIS platform and carries out an evacuation simulation. Then, the information on the induction for evacuation is sent to a local area or to a local core facility through the DiMIS platform (Figure 6b).

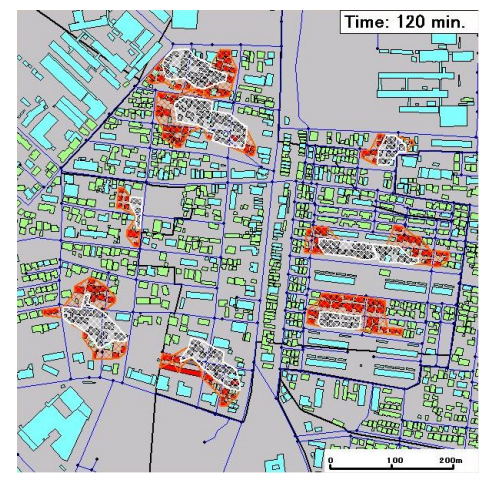

(a) Fire spreading simulation

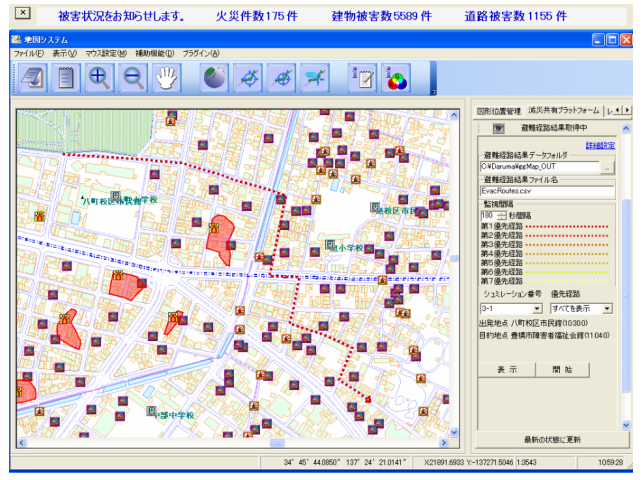

(b) Evacuation simulation

Figure 6: Screen shots of the simulation system. 


\section{Field test on the application of the DiMIS platform to a local government}

\subsection{Purpose and field test}

As described above, the DiMIS platform is composed of various information systems. The verification of each system does not necessarily mean the verification of the whole system. In order to verify that the platform is effective to disaster response activities, therefore, a prototype of the platform was built by the integration of different systems and tools developed in this research project, and it was applied to a local government.

Mitsuke city was selected as the field test. The city, with the population of about 44000, is located at a central area of Niigata prefecture, featuring a rural district with rice fields. The city is divided into north and south by the Kariyata river, which repeatedly caused floods in the past. Mitsuke city suffered two notable disasters, i.e., the Fukushima-Niigata heavy rainfall of 2004 and the central Niigata prefecture earthquake of 2004. Local government employees of Mitsuke city are well trained by disaster responses in actual disasters, as well as being highly conscious of disaster mitigation and prevention, since they have experience these disasters. Thus, they can take part in the field test as players and can evaluate the effectiveness of the platform to disaster response activities in local governments.

\subsection{Method of the field test}

The field test is consisted of 6 parts as shown in Table 1. The heavy rainfall was selected as a disaster phenomenon adopted in the test. In each part, issues for verification were set up and scenarios were written. Nine different research

Table 1: $\quad$ Description of the field test.

\begin{tabular}{|c|l|l|}
\hline No. & \multicolumn{1}{|c|}{ Title of each part } & \multicolumn{1}{|c|}{ Organization evaluating the test } \\
\hline 1 & Information and establishment & Mitsuke city \\
\hline 2 & $\begin{array}{l}\text { Collection of disaster } \\
\text { information and disaster } \\
\text { response (drill) }\end{array}$ & $\begin{array}{l}\text { Mitsuke city, Niigata prefecture, } \\
\text { Fire and Disaster Management } \\
\text { Agency and Cabinet Office }\end{array}$ \\
\hline 3 & Management of place of refuge & Mitsuke city \\
\hline 4 & Induction of evacuation & $\begin{array}{l}\text { Lifeline utilities, Police station of } \\
\text { Mitsuke, Niigata prefecture, Fire } \\
\text { and Disaster Management Agency }\end{array}$ \\
\hline 5 & $\begin{array}{l}\text { Distribution of information to } \\
\text { the mass media }\end{array}$ & $\begin{array}{l}\text { Mitsuke city and the mass } \\
\text { communication media participated } \\
\text { in the test }\end{array}$ \\
\hline 6 & $\begin{array}{l}\text { Information sharing among } \\
\text { various organizations }\end{array}$ & $\begin{array}{l}\text { Cabinet Office, Fire and Disaster } \\
\text { Management Agency, Mitsuke city, } \\
\text { Niigata prefecture, Lifeline utilities, } \\
\text { Police station of Mitsuke }\end{array}$ \\
\hline
\end{tabular}


institutions out of 13 took part in the field test. In addition, 9 different organizations related to the disaster response activities of Mitsuke city joined the test as collaborators. Therefore, a number of researchers and disaster prevention personnel and government officials participated in the field test.

In the second part of the field test, the disaster response drill was executed by local government employees of Mitsuke city as players in the circumstances of the DiMIS platform. They collected disaster information and responded using the disaster response management system. After the drill, they evaluated the effectiveness of the platform compared the response activities between with and without the platform in dependence upon their experience. In other parts of the field test, the collaborators only evaluated the effectiveness of the platform right after they observed the demonstration conducted by the research institutions.

The field test was mainly conducted in Mitsuke city. Some data are transmitted to Niigata prefecture government in Niigata city and the Fire and Disaster Management Agency in Tokyo, through the public broadband network of the Ministry of Internal Affairs and Communication.

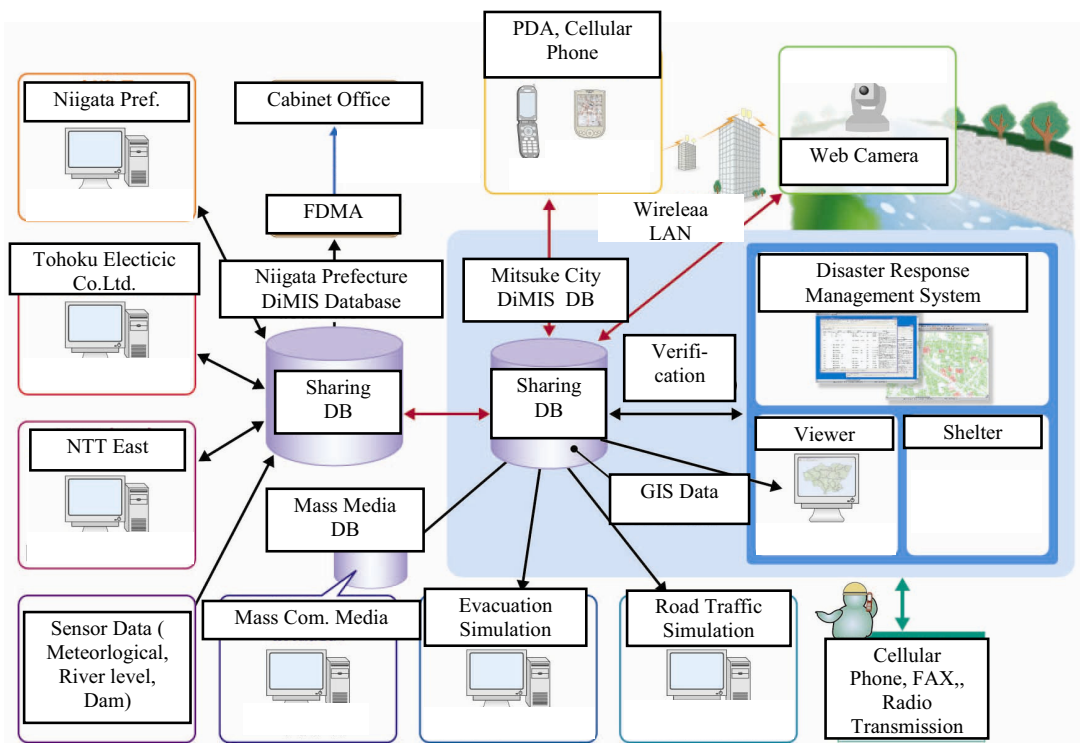

Figure 7: The DiMIS platform applied to the field test.

\subsection{Platform applied to the field test}

Figure 7 illustrates a schematic diagram to describe the prototype of the DiMIS platform constructed for the field test. The DiMIS database was allocated inside and outside Mitsuke city, respectively. The database inside Mitsuke city is the information sharing database server for Mitsuke city and it was connected with such client systems as the disaster response management system, simulators, information collection systems (PDA, cellular phone) and a viewer system. The viewer system is the system to demonstrate contents of information in the 
database. In addition, the database is connected with the information sharing database outside the city (Niigata Prefecture) and the database for mass communication media.

The DiMIS database outside Mitsuke city is supposed to be located in Niigata Prefecture Government. It is connected with information sharing systems of lifeline utilities such as Tohoku Electric Power Co., Ltd. and NTT East, Niigata prefecture (including the river and dam information center), and the Fire and Disaster Management Agency in Tokyo.

Both databases inside and outside Mitsuke city were connected. The database in Niigata prefecture transmits information from Niigata prefecture and lifeline utilities to Mitsuke city and receives information of disaster damages, disaster response activities and requests from Mitsuke city. Such information sharing between the two databases is executed automatically by polling action.

\subsection{Outline of the test results}

The field test was carried out mainly in the meeting room in the city hall of Mitsuke city where the headquarters of disaster countermeasure was set up. Government officials of the Fire and Disaster Management Agency observed the field test at the risk management center in Tokyo.

The second part of the test was carried out as a disaster response drill as mentioned, several local government employees were sent to outside the city hall for the investigation of disaster damages. The damage was sent to the DiMIS platform using PDA, cellular phones and shared through a viewer (Figure 8) as well as on screens of the disaster response management system (Figure 9).

Figure 10 shows a screen shot superimposing lifeline information over geographical and damage information. Each area of blackout of power supply and telecommunication, closed roads and flood areas respectively was superimposed on the geographical information. Such superimposition of data can be made onto Google Earth (Figure 11).

After finishing the final part of the test, the evaluation of the platform was made by collaborators in the form of both questionnaire and interview. The platform was evaluated as highly effective to disaster response activities. In his closing remarks, Mr. Kusumi, Mayer of Mitsuke city expressed his gratitude to us and indicated that he wanted to introduce the platform to Mitsuke city for the safe of citizens.

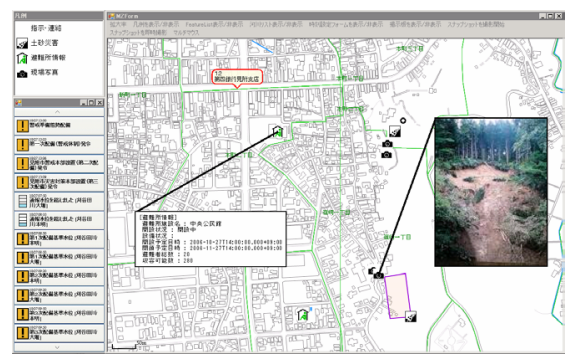

Figure 8: Viewer system.

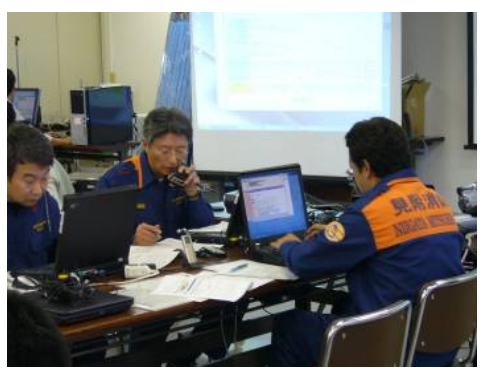

Figure 9: Disaster response (Part 2). 


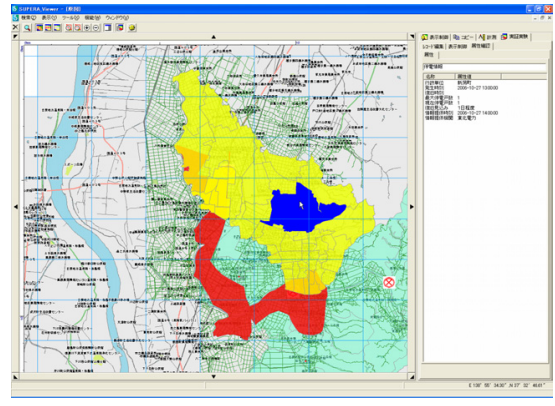

Figure 10: Superposition of lifeline information (Part 6).

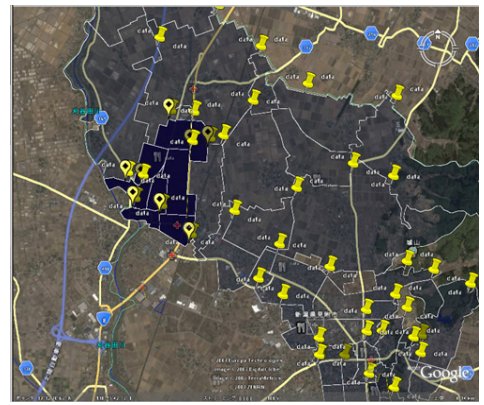

Figure 11: Superimposition of various information on Google Earth.

\section{Concluding remarks}

This paper presented the outline of the three-year joint research project on the disaster mitigating information sharing platform. The framework of the platform was established and various information systems and tools were developed as a prototype of the platform. The prototype was applied to the field test. Then, the platform was evaluated as highly effective to disaster response activities.

Researchers who participated in this project have established NPO named as ADMiRe (Agency for Promoting Disaster Mitigation and Damage Reduction) in order to realize the platform and started their activities in various fields.

\section{References}

[1] Suzuki, T. and Goto, Y., Introduction of an approach to disaster mitigation using crisis-adaptive information sharing platform and technology, Risk Analysis, V, pp.119-125, 2006.

[2] Suzuki, T. and Hada, Y., Joint research project on disaster reduction using information sharing technologies, Proceeding of the $2^{\text {nd }}$ International Conference on Urban Disaster Reduction, Taiwan, 2007 (accepted).

[3] Suzuki, T. and Goto, Y.: Framework on disaster mitigating information sharing platform, Proceedings of the $12^{\text {th }}$ Japan Earthquake Engineering Symposium, pp.1346-1349, 2006 (in Japanese).

[4] Suzuki, T. and Amami, M.: Investigation on actual disaster responses in local governments in terms of information sharing, Proceedings of Japan Society of Disaster Information Studies (submitted).

[5] Suzuki, T. and Amami, M.: Disaster response management system for local government and its application to the response drill, Proceedings of Earthquake Engineering, JSCE, Vol.29, pp.71-790, 2007 (in Japanese).

[6] Hada, Y., Suetomi, I., Suzuki, T. and Nabana, K.: Lifeline information sharing through the disaster mitigating information sharing platform, Proceedings of the $12^{\text {th }}$ Japan Earthquake Engineering Symposium, pp.1342-1345, 2006 (in Japanese). 